\title{
WSN-based near Real-time Environmental Monitoring for Shelf Life Prediction through Data Processing to Improve Food Safety and Certification
}

\author{
G. E. Biccario, V. F. Annese, S. Cipriani and D. De Venuto \\ Department of Electric and Informatic Engineering - DEI, Politecnico di Bari, Via Orabona 4, Bari, 70125, Italy
}

\begin{abstract}
Keywords: Environmental Control, Food Safety, Food Certification, Shelf Life Prediction, Wireless Sensor Network.
Abstract: This position paper aims to support a control technique in the perishables goods supply-chain through a combination of near real-time wireless sensor network (WSN) for environmental monitoring and further data processing to predict the shelf life of the product. This approach returns a low cost, versatile and efficient tool that can significantly improve the safety and food certification through the organoleptic qualities control using three different sensors, i.e. temperature, light and humidity. In this article, therefore, the advantages of the proposed technique are explained and a case study is presented to support this approach, as well as an example of processing algorithm for shelf life evaluation.
\end{abstract}

\section{INTRODUCTION}

Nowadays, food safety and certification through-out all the supply chain have become very strict requirements, so that global associations such as the FDA ("Food and drug administration") or the WHO ("World Health Organization") are continuously in activity to develop and promote more selective methods of monitoring and control (FAO/WHO, 2007). Organoleptic properties of food, raw food, cosmetics, drugs, specialty oral use and other perishable products, affect considerably not only the safety of the product for human use but also its consumption and commercial success. Hence the need to study, define and evaluate them correctly in order to prevent their earlier degradation. In fact, the loss of perishable products is estimated at approx. $\$ 35$ billion annually all around the world and especially in US (Hoppough, Apr. 24, 2006).

A multitude of security and certification protocols has been developed and spread in all areas of the food supply chain and, among all, the "Hazard analysis and critical control points" (HACCP) method continues to be worldwide increasingly prevalent. HACCP is a scientific and technical approach for the prevention of biological, chemical, and physical hazards, whose seven fundamental principles are released and determined by the standard ISO 22000 (ISO, 2005).

The HACCP aim is to identify the different critical control points (CCPs) in food production and to define the parameters of interest for suitably monitoring every phase of the productive process. This approach creates a major emphasis on food quality, particularly with regard to health and safety, a concept that goes beyond mere customer satisfaction, but rather pointing to the protection of public health (FAO/WHO, 2007).

An "ad hoc" WSN is an effective solution for monitoring perishable goods during all their chain supply. The near real time data availability, the low power consumption, the possibility of expand the WSN using the most appropriate components for each application, the moderation of the production costs and the adequate precision of the measures are all great reasons that make a WSN an advantageous solution in perishable food supply chain. The gathered data can be used to prevent not recommended environmental conditions, in particular during the storage and distribution phases, according to HACCP-like methods, in order to prevent product rapid decay and, therefore, losses. Moreover, they can be used to evaluate product freshness via shelf-life (Labuza, 2001) parameter, which is the period of time in which a perishable product is compliant with declared nutritional data values and sensory, chemical, physical and microbiological characteristics, without becoming unhealthy for use or consumption, when stored under well-defined conditions (Institute of Food 
Science and Technology, 1993). In this paper, a WSN-based environmental monitoring is described as a case study to prove the effectiveness of this approach. An algorithm for later data processing is proposed for the near real-time availability of shelflife evaluation.

The paper is organized as follows: sections 2 and 3 propose related works and WSN architecture, including (in section 4) a demonstrative monitoring of three storage conditions affecting the organoleptic properties of perishable goods, i.e. temperature, relative humidity and light exposition, as case study. The measurements have been realized in a warehouse for storage of agricultural products. The section 5 shows an algorithm for the processing of the obtained data: a shelf life estimation through a calculation algorithm based on the Arrhenius law. The paragraph 6 shows an attempt of financial statement.

\section{RELATED WORKS}

A Wireless sensor network (WSN) is an infrastructure composed by wireless nodes, with little memory and a low-performance CPUs, capable of performing measurements, processing and communicating wirelessly to a central point, where the data are managed (Sohraby, 2007). The structure typically involves several wireless scattered nodes in a specific area periodically sending the collected data to a coordinator point (gateway), which manages the network and forwards them to another remote system for further processing (Dargie, 2010). The wireless access is usually a "contention-oriented random access" type, as defined in the IEEE 802, but IEEE 802.15.4 is the most commonly used standard, in particular ZigBee standard. This is due to IEEE 802.15.4 applicative advantages like worldwide defined operative band (2.4 GHz ISM), good data rate $(250 \mathrm{kbps})$ and range of action (several tens of meters), low power consumption, possibility of routing and retransmission in case of errors (Lee, 2007).

WSN effectiveness has been proved in many applications concerning agro-food context (Yoo, 2007). In (Garcia-Sanchez, 2011) ZigBee-based WSN is used for data-monitoring and video surveillance in precision agriculture over distributed crops. Other examples of monitoring during the production phase are precision viticulture, as presented in (Matese, 2009), and greenhouse control (Dae-Heon Park, 2011). As concern storage monitoring, WSN solution has been employed in grain warehouse (Zhao, 2010) and fresh food supermarket (Yang Chenwei, 2011). As described in (Ko, 2014), WSN have proved to be an excellent solution for real time traceability and monitoring of agricultural product.

Although WSNs have already been effectively employed in environment monitoring from production to distribution phases, so that gathered data can be used to generate alarms when specific events are detected (Dennis J. A. Bijwaard, 2011), they are not supported by a shelf-life evaluation, which would ensure product adequate quality along the supply chain.

In this paper a WSN-based monitoring, supported by shelf-life valuation from environmental variables, is presented; the collected data are used in a simple degradation model to calculate shelf-life which is communicate to the users through an algorithm and can be used to send alarms when recommended conditions are not verified anymore.

\section{$3^{\text {WIRELESS SENSOR }}$ NETWORK ARCHITECTURE}

The WSN used for the monitoring activity consists of several nodes equipped with three different sensors to obtain information about the environmental light, temperature and humidity (figure 1). Each unit sends data to a coordinator whose task is to make them available in almost real time (neglecting the communication delays). The communication between each node and gateway is wireless using Zigbee protocol. Subsequently, the gateway uploads the data to a cloud using an internet connection. The data are available on the web using a tablet, a smartphone, a PC or any device with internet connectivity by logging in the data cloud

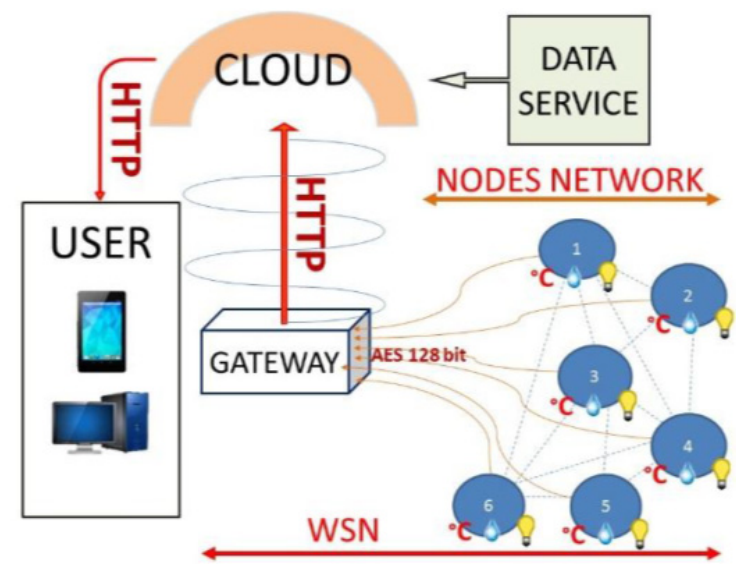

Figure 1: Architecture of the employed WSN. 
that manages the data. An appropriate authentication system allows access only to authorized users.

In figure 2 there is a demonstrative diagram about the overall architecture of a single node. The small size $(6.85 \mathrm{~cm} \times 6.35 \mathrm{~cm} \times 3.30 \mathrm{~cm})$ and the independent power supplyallow proper positioning of the sensors. Due to the use of the Zigbee technology and to its low power consumption, the battery life is durable.

Table 1 summarizes the main performance of each node. The analog outputs of the sensors are digitized by a 12 bit resolution $\mathrm{ADC}$ and then are

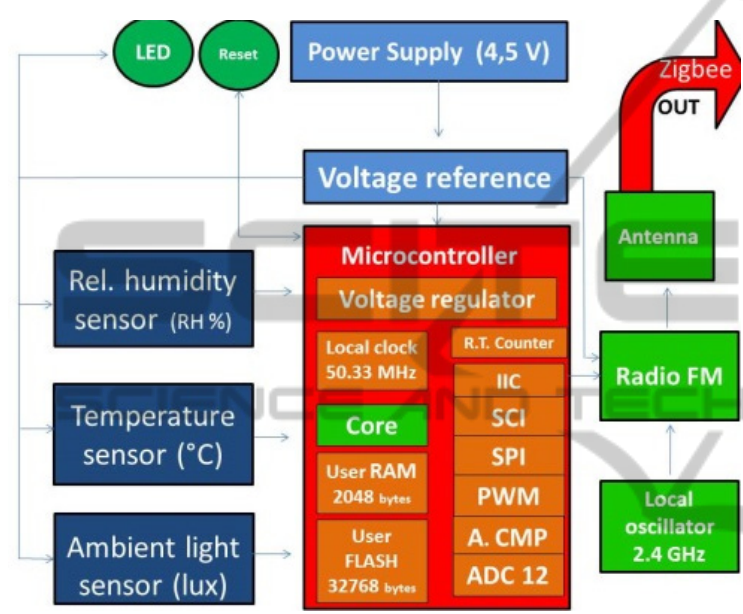

Figure 2: Architecture of a node of the WSN.

Table 1: Node sensor performances.

\begin{tabular}{|c|c|c|}
\hline $\begin{array}{l}\text { Power } \\
\text { supply }\end{array}$ & $\begin{array}{l}\text { Specification } \\
\text { Read-sleep } \\
\text { cycle/ battery } \\
\text { life } \\
\text { AC input V }\end{array}$ & $\begin{array}{c}\text { Value } \\
1 \text { per } 30 \mathrm{~s} / 1.5 \mathrm{y} \\
1 \text { per } 60 \mathrm{~s} / 2.5 \mathrm{y} \\
\begin{array}{c}3 \mathrm{x} \text { AA } 1.5 \mathrm{~V} \\
\text { battery }\end{array}\end{array}$ \\
\hline $\begin{array}{l}\text { Temp. } \\
\text { sensor }\end{array}$ & $\begin{array}{c}\text { Range } \\
\text { Accuracy }\end{array}$ & $\begin{array}{c}-18 \text { to }+55^{\circ} \mathrm{C} \\
+/-2\end{array}$ \\
\hline $\begin{array}{c}\text { Ambient } \\
\text { light } \\
\text { sensor }\end{array}$ & $\begin{array}{c}\text { Bandwidth } \\
\text { Range } \\
\text { Wavelength of } \\
\text { peak sensitivity } \\
\text { Luminance } \\
\text { range }\end{array}$ & $\begin{array}{l}360 \text { to } 970 \mathrm{~nm} \\
570 \mathrm{~nm} \\
10 \text { to } 1000 \mathrm{lux} \\
(+/-20 \%)\end{array}$ \\
\hline $\begin{array}{l}\text { Relative } \\
\text { humidity } \\
\text { sensor }\end{array}$ & $\begin{array}{c}\text { Range } \\
\text { Interchangeabil } \\
\text { ity }\end{array}$ & $\begin{array}{c}0 \text { to } 95 \% \mathrm{RH} \\
+/-5 \%(0 \text { to } 59 \% \\
\mathrm{RH}) \\
+/-8 \%(60 \text { to } 95 \% \\
\mathrm{RH}) \\
+/-3.5 \% \mathrm{RH}\end{array}$ \\
\hline $\begin{array}{c}\text { Zigbee } \\
\text { transmiss } \\
\quad \text { ion }\end{array}$ & $\begin{array}{l}\text { RF date rate } \\
\text { Frequency } \\
\text { Indoor/line of } \\
\text { sight range }\end{array}$ & $\begin{array}{c}\text { 250kbps } \\
\text { ISM } 2.4 \mathrm{GHz} \\
40 \mathrm{~m} / 120 \mathrm{~m}\end{array}$ \\
\hline
\end{tabular}

processed for transmission. sensor specifications should match the requirements of the product they are intended to monitor. since this wsn has a demonstrative purpose their values have to be considered suggestive.

\section{WSN IN A PERISHABLES GOODS SUPPLY-CHAIN: EXPERIMENTAL RESULTS}

In this paragraph a case study is described to demonstrate the feasibility and effectiveness of the proposed solution. An "ad-hoc" solution requires an adequate choice of the smart sensor selected once inside the "working" environment and a positioning and mapping phase of the nodes to get the traceability of the product.

\subsection{Sensor Performance Analysis}

A preliminary performance analysis of the nodes had

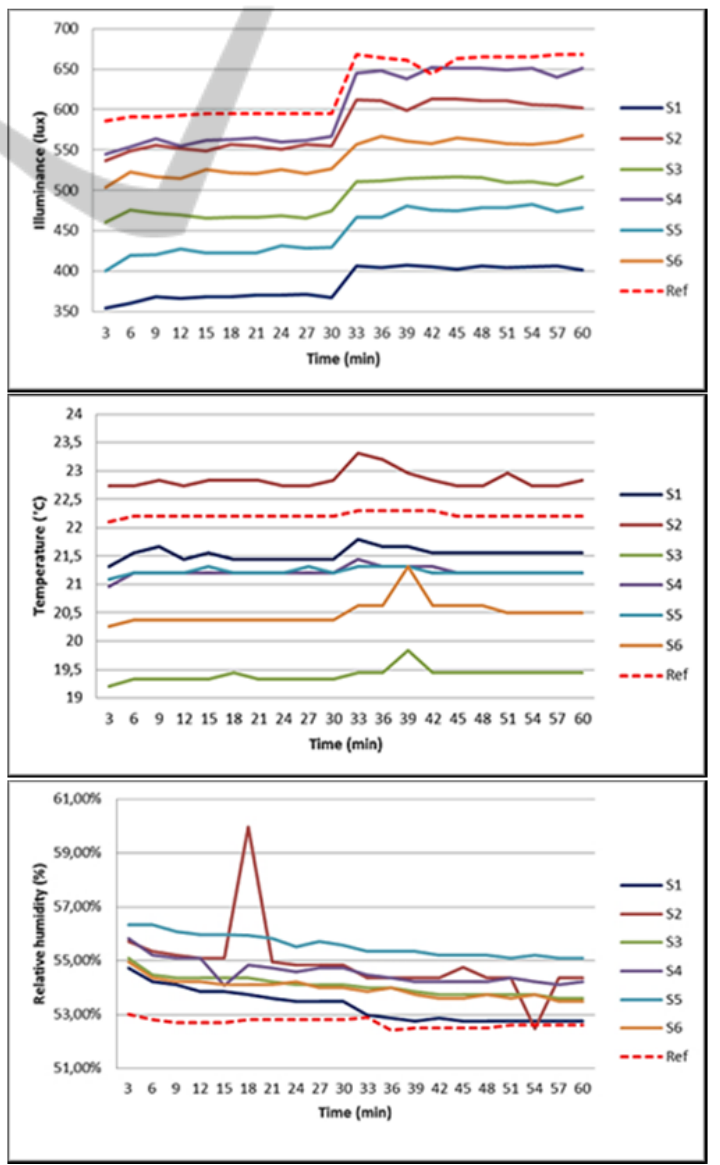

Figure 3: from the top: illuminance, temperature and relative humidity values collected during sensor accuracy analysis and compared to the reference ones. 
been performed: using comparative reference, sensors accuracy have been evaluated. During one hour session20 samples have been collected and compared with those of more accurate instruments (hygrometer-thermometer: Testo 608-H1; luxmeter: Yokogawa 510 O2).

Measurements were carried out indoor with artificial lights. The obtained values are shown in figure 3 , where the red dashed line represents the values given by the instrument used as reference.

Sensors average values (table 2) have been compared with reference average value to calculate accuracy; standard deviation from average values is useful to evaluate sensors precision. Sensors performances are sufficient for an environmental monitoring which therefore does not require extreme precision.

Table 2 Illuminance, temperature and relative humidity sensor averages and standard deviation for accuracy and precision evaluation.

\begin{tabular}{ccc}
\hline & Average [lux] & Std. Dev. [lux] \\
S1 & 385.6 & 19.78 \\
S2 & 580.05 & 28.73 \\
S3 & 491.05 & 22.48 \\
S4 & 603.65 & 44.30 \\
S5 & 449.45 & 27.47 \\
S6 & 540.75 & 21.24 \\
Ref. & 628.1 & - \\
\hline & Average $\left[{ }^{\circ} \mathbf{C}\right]$ & Std. Dev. $\left[{ }^{\circ} \mathbf{C}\right]$ \\
S1 & 21.54 & 0.11 \\
S2 & 22.84 & 0.16 \\
S3 & 19.05 & 0.12 \\
S4 & 21.21 & 0.086 \\
S5 & 21.22 & 0.06 \\
S6 & 20.50 & 0.22 \\
Ref. & 22,21 & - \\
\hline & Average [rh\%] & Std. Dev. $[\mathbf{r h \%} \%$ \\
S1 & 53.32 & 0.0060 \\
S2 & 54.89 & 0.0132 \\
S3 & 54.06 & 0.0036 \\
S4 & 54.57 & 0.0045 \\
S5 & 55.56 & 0.0041 \\
S6 & 53.95 & 0.0035 \\
Ref. & $\mathbf{5 2 . 6 8}$ & - \\
\hline
\end{tabular}

\subsection{WSN-based Monitoring of a Dehydrated Agricultural Product Warehouse}

The presented WSN has been employed for the monitoring of a warehouse of dehydrated agricultural products. Three multi-sensor nodes have been used to monitor three different pallets in the same environment. Figure 4 shows collected data values of the temperature, relative humidity and light exposition during a 5 hours monitoring, from $10: 17$ to $15: 17$; three values per second were collected and then averaged to reduce data throughput. One node was placed in an indoor pallet (blue curve), the others out of the pallet but in the warehouse. Thanks to the battery life durability, it would be possible a monitoring of the entire food chain it in the same way. However, sampling data could be significantly reduced to increase node lives. This would promote transparency in the food chain, becoming a guarantee for the consumer, a powerful low-cost tool for the producer and a simple control method for the predisposed organs. The collected data became available to any authorized user in the world almost in real time, neglecting a little latency due to communication (few tens of ms).

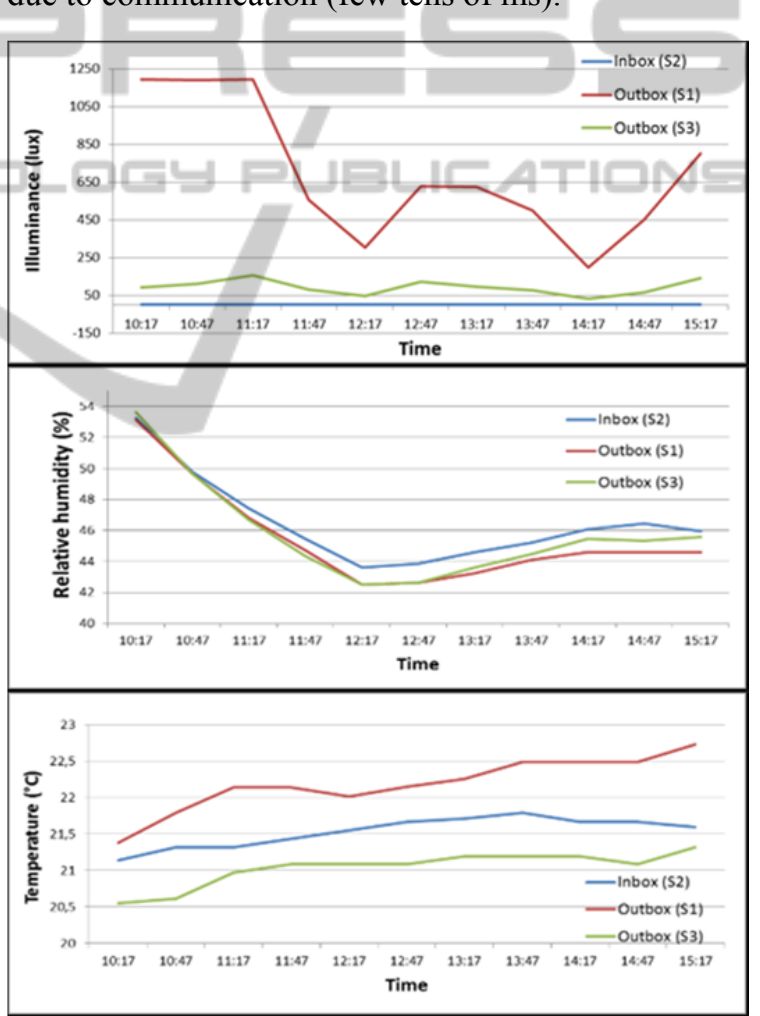

Figure 4: from the top, illuminance (lux), relative humidity (\%) and temperature $\left({ }^{\circ} \mathrm{C}\right)$ values collected during the monitoring of a dehydrated agricultural products.

\section{SHELF LIFE ESTIMATION FROM QUALITY DEGRADATION RATE}

Shelf-life evaluation depends on the nature of the 
considered product and, thus, on the environmental factors involved in its degradation. Temperature, light exposition and humidity affect product quality at the same time but as concern the products in the presented study, the implementation of Arrhenius and Lambert law in a calculation algorithm has been considered to estimate the degradation rate of the agricultural products, and thus their shelf life, assuming that recommended characteristics are known.

\subsection{Linear Mathematical Model of Quality Degradation Prediction}

The relationship between product's quality and time is considered approximately linear (Figure 5): $C$ stands for the food quality $\left(C_{o}\right.$ : recommended condition; $C_{e}$ : minimum quality level), $t_{s}$ for the shelf time, $K$ for the quality degradation speed. $\mathrm{K}$ depends on the temperature according to Arrhenius law [4]

$$
\equiv \square I \equiv \frac{d c}{d t}=K(T)=W e^{\frac{-E a}{R T}}
$$

$C_{o}, C_{e}, t_{S}, T_{0}$, the recommended storage temperature, have to be considered known (reference situation). So $K_{O}$, the reference degradation speed is given by:

$$
K_{o}=\frac{C_{e}-C_{o}}{t_{s}}
$$

If at time $t_{1}$ the temperature rises up to $T_{1}>T_{O}$, above a certain threshold, it's possible to estimate $K_{1}$ (the new degradation speed) using eq. (1). The new characteristic can be expressed as:

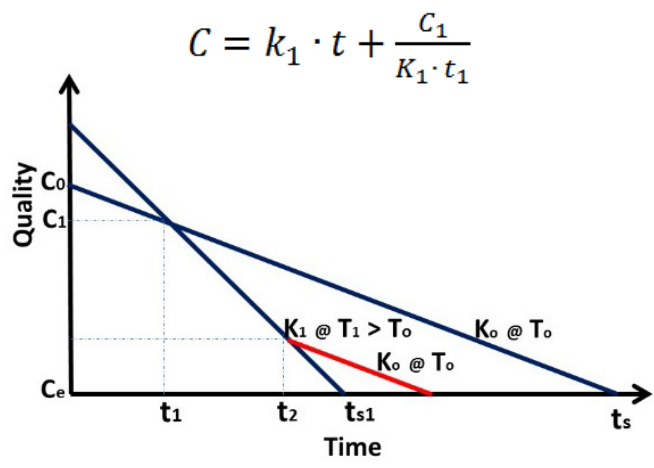

Figure 5: Linear model of quality degradation.

The new perspective produces a lower shelf-time $t_{S 1}$. If the temperature returns from $T_{1}$ to $T_{o}$, the shelf life must not change: by now the product quality has been spoiled.

\subsection{Algorithm for Shelf Life Prediction}

Similarly to (Azanha, 2005), an algorithm for shelf life prediction is proposed, according to the model described above. The purpose is to create an application for smartphone and tablet which is able to calculate in real time how long the product preserve the quality specifications in that environment.

I. Store input data about standard shelf life (SLo [h]), temperature (To), Humidity (Rho) and illuminance (Lxo) of recommended value. Acquire from the WSN information about current T, Rh, Lx, than show them.

II. Verify if the current conditions are above certain thresholds from the reference ones (the chosen range is due to sensors performances). If not, calculate the $K_{O}$. The $\mathrm{SL}$ is the same as the reference SL (stored in input).

III. If the product is not in the standard condition, create an alert signal. Than calculate the new degradation speed coefficient $(\mathrm{K})$ through Arrhenius equation (1) and estimate the new shelf life as a fraction of the standard one: the new shelf time is calculated using the ratio between the current degradation speed and the previous one. In order to perform these calculation, a database with all the required constant values is needed. Finally set the new SL as a optimistic value: the product has been spoiled (in a preventive view) and the SL cannot be restored (even if returning in the recommended conditions).

IV. Show remaining shelf time and repeat the loop every hour.

\subsection{Accuracy of Shelf-Life Prediction}

The calculation system proposed exhibits a certain degree of approximation. Firstly, the calculation is made assuming the degradation as a linear function of time although in reality it is not. Moreover, considering that the temperature sensors have an accuracy of $+/-2^{\circ} \mathrm{C}$, we can reasonably assume that the new value of the rate of degradation $K_{1}$ is to be understood between a $K_{1-}$ and $K_{1+}$, evaluated according to the Arrhenius law (equation (1)) at $T_{1}+2{ }^{\circ} \mathrm{C}$ and $T_{1}-2{ }^{\circ} \mathrm{C}$ respectively. Therefore the shelf life is valid in an interval between $t_{S 1-}$ and $t_{S 1+}$, according to eq. (3), using respectively $K_{1-}$ and $K_{1+}$.

The accuracy of the system can surely be improved by using more accurate sensors for the measurement of the temperature and implementing a method of calculation based on a more specific 
mathematical (even better if the model is differentiated by class of foods).

\section{FINANCIAL CONSIDERATION: COSTS AND BENEFITS}

The proposed solution is not expensive and allows a safe monitoring of several typology of goods. Just for give an estimation of the costs let's try to make a budget statement. If produced in a supply chain, we can estimate the cost of each node of about $€ 15$ (considering nodes of high quality with reprogrammability and reusability characteristics) and about $€ 500$ for each gateway. A system made by 3 gateways and 45 sensors (considered sufficient to manage a medium-sized productive environment) would cost about $€ 2,175$. Estimating the lifetime of the gateway (changing individual nodes is not a problem) for about 4 years, we are talking about $€$ 544 annually. Regarding the shelf life estimation and the implementation of the dedicated application, we can assume that in a supply chain cost is negligible if compared to the WSN one. These costs would certainly be overcome by the consequent reduction of wastage in perishables chain and the corresponding increase in sales (due to the added value that such a monitoring system can provide the product).

\section{CONCLUSIONS}

Safety and certification for food production is not an objectionable topic but a strong need: WSN can be used as an effective tool to allow both a reduction of the waste in the supply chain through corrective actions when recommended conditions are not satisfied anymore and an improvement of food safety through shelf-life estimation. A case study of warehouse monitoring with a WSN architecture has been described to support the feasibility and low cost characteristic of this solution; three environmental conditions, affecting the organoleptic properties of perishable goods, i.e. temperature, humidity and light exposition have been used in a proposed algorithm, based on the Arrhenius law, to estimate shelf life. Our work was therefore just a demonstration of feasibility, but future prospects are even more persuasive thanks to the several application fields (not only agri-food) and the flexibility of WSN architectures.

\section{REFERENCES}

Azanha, A., 2005. Use of mathematical models for estimating the shelf-life of cornflakes in flexible packaging. Packaging Technology and Science, 18(4), pp. 171-178.

Dae-Heon Park, e. a., 2011. A Study on Greenhouse Automatic Control System Based on Wireless Sensor Network. Wireless Pers Commun, Volume 56, pp. 117-130.

Dargie, W. a. P., 2010. Fundamentals of wireless sensor networks: theory and practice. s.1.:C.John Wiley and Sons.

Dennis J. A. Bijwaard, e. a., 2011. Industry: using dynamic WSNs in smart logistics for fruits and pharmacy. Seattle, USA, Proceedings of the 9th ACM Conference on Embedded Networked Sensor Systems, pp. 218-213.

FAO/WHO, 2007. FAO/WHO guidance to governments on the application of HACCP in small and/or lessdeveloped food businesses. s.1.:s.n.

Garcia-Sanchez, A. J., 2011. Wireless sensor network deployment for integrating video-surveillance and data-monitoring in precision agriculture over distributed crops. Computers and Electronics in Agriculture, 72(2), pp. 288-303.

Hoppough, S., Apr. 24, 2006. Shelf life. Forbes.

Institute of Food Science and Technology, 1993. Shelf-life of foods: guidelines for its determination and prediction. London: s.n.

ISO, 2005. ISO22000, Food Safety Management System. s.1.:s.n.

Ko, D., 2014. Real Time Traceability and Monitoring System for Agricultural Products Based on Wireless Sensor Network. International Journal of Distributed Sensor Networks, Volume 2014.

Labuza, T. P. S. L., 2001. Open dating of Foods. II ed. Trumbull, Connecticut, USA: Food and Nutrition Press.

Lee, J., 2007. A Comparative Study of Wireless Protocols: Bluetooth, UWB, ZigBee, and Wi-Fi. Taipei, Taiwan, s.n.

Matese, A. D. G. S. F., 2009. A wireless sensor network for precision viticulture: the NAV system. Computer and Electronics in agricolture, 69(1), pp. 51-58.

Sohraby, K., 2007. Wireless sensor networks: technology, protocols, and applications. Hoboken, New Jersey: John Wiley and Sons.

Yang Chenwei, L. D. Y. D. Y. S. L. Z., 2011. Design of Monitor-and-control System for Supermarket Fresh Area Based on ZigBee. Wuhan, IEEE, pp. 1302-1305.

Yoo, S., 2007. A2S: Automated agriculture system based on WSN. Irving, ISCE 2007. IEEE International Symposium on Consumer Electronics.

Zhao, Y., 2010. The grain depot temperature measurement system's research based on wireless sensor networks. Jinan: IEEE. 